\title{
Nanotheranostics
}

2019; 3(1): 54-65. doi: 10.7150/ntno.27452

Research Paper

\section{Comparing Semiconductor Nanocrystal Toxicity in Pregnant Mice and Non-Human Primates}

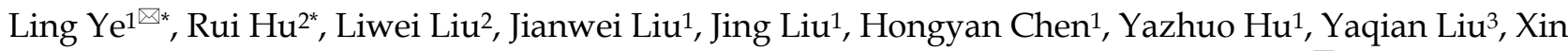 \\ $\mathrm{Liu}^{4}$, Cui Liu ${ }^{4}$, Danny Jian Hang Tng'5, Yuanguang Meng6, Junle Qu², Mark T. Swihart ${ }^{7 凶}$, and Ken-Tye \\ Yong8 \\ 1. Institute of Gerontology and Geriatrics and Beijing Key Lab of Aging and Geriatrics, Chinese PLA General Hospital, Beijing 100853, P. R. China \\ 2. Key Laboratory of Optoelectronic Devices and Systems of Ministry of Education and Guangdong Province, College of Optoelectronic Engineering, \\ Shenzhen University, Shenzhen 518060, P. R. China \\ 3. Laboratory Animal Center, Chinese PLA General Hospital, Beijing 100853, P. R. China \\ 4. Department of Nan-Lou Ultrasound, Chinese PLA General Hospital, Beijing 100853, P.R. China \\ 5. Duke-NUS Medical School, Singapore 169857, Singapore \\ 6. Department of Obstetrics and Gynecology, Chinese PLA General Hospital, Beijing 100853, P. R. China \\ 7. Department of Chemical and Biological Engineering, University at Buffalo, USA \\ 8. School of Electrical and Electronic Engineering, Nanyang Technological University, Singapore 639798, Singapore \\ "These authors contributed equally to this work \\ $\square$ Corresponding authors: Ling Ye, Mark T. Swihart, Ken-Tye Yong \\ (c) Ivyspring International Publisher. This is an open access article distributed under the terms of the Creative Commons Attribution (CC BY-NC) license \\ (https://creativecommons.org/licenses/by-nc/4.0/). See http://ivyspring.com/terms for full terms and conditions.
}

Received: 2018.05.24; Accepted: 2018.10.27; Published: 2019.01.01

\begin{abstract}
Rationale: Despite growing use of engineered nanomaterials (ENM) in applications from electronics to medicine, the potential risk to human health remains a critical concern within clinical use. ENM exposure during pregnancy can potentially cause reproductive toxicity even at levels that produce no measurable harm to animals in normal conditions.
\end{abstract}

Methods: Phospholipid micelle-encapsulated $\mathrm{CdSe} / \mathrm{CdS} / \mathrm{ZnS}$ semiconductor nanocrystals with an average hydrodynamic diameter of $60 \mathrm{~nm}$ were intravenously injected during pregnancy in both rodent and nonhuman primate animal models. Cadmium concentration levels and maternal haematological and biochemical markers were determined, along with histopathological examination of major organs.

Results: Nanocrystals were found to have crossed the placenta from mother to fetus in both rodents and nonhuman primates. However, the animal models display different responses with respect to reproductive toxicity. In the rodent model, toxicity symptoms are absent in treated subjects, with no observed gestational or fetal abnormalities and complications. A significantly higher miscarriage rate of $60 \%$ is recorded for macaques after prenatal nanoparticle administration. There was a miscarriage rate of $15 \%$ in the general population despite only $~ 0.16 \%$ of the initial cadmium dose present in the fetus. Blood and biochemical markers of treated macaques indicate acute hepatocellular injury within a week after nanoparticle administration. Histology of major organs of the miscarried macaque fetuses show no abnormalities.

Conclusion: The potential of nanomaterials to cross the placenta and impact fetal survival in primates suggest the necessity of precautionary measures to prevent gestational exposure of ENMs.

Key words: quantum dots, nanotoxicology, biophotonics, engineered nanomaterials

\section{Introduction}

Worldwide production of semiconductor nanoparticles, often called quantum dots (QDs), is on the rise, with a total annual production now measured in tons. The global market for semiconductor 
nanoparticles has reached $\$ 610.0$ million in revenues in 2017 and is projected to grow at a compound annual growth rate of $41.3 \%$ while having an estimation to reach about $\$ 3.4$ billion by 2021 [1]. Along with economic and technological benefits, this rapid growth of using semiconductor nanoparticles raises concerns on environmental and health effects stemming from prolonged exposure to semiconductor nanoparticle-containing products in our daily life [2, $3]$. In order to develop new strategies for human safety in this nanoparticle era, detailed and complete toxicity assessment in relation to the engineered nanomaterials (ENMs) is required [4]. Among women of reproductive age, exposure to nanoparticles is increasingly prevalent especially as these nanoparticles are found in the environment and various commercial products such as white light LEDs and cosmetics [1]. It is reported that a substantial fraction of infants worldwide manifested certain characteristics of chronic disorders that resulted from maternal exposure to toxic chemicals during pregnancy [5]. The rapid growth of the ENM productions have blended and became part of our daily life which have made it a necessity for more attention to be paid on their potential risks towards the reproductive system and pregnancy [6]. Recent studies have shown that nanoparticles could migrate across the placenta in pregnant small animals and caused adverse effects to their offspring [7]. For instance, Chu et al. have shown that QDs may be transferred from female mice to their fetuses across the placental barrier and the transplacental behavior is dependent on the particle size and dosage. They have also revealed that surface modification with an inorganic silica shell or organic polyethylene glycol could reduce the QD transfer yet does not eliminate it [8]. Wang et al. have shown that low-dose chronic exposure to QDs before pregnancy could result in QD accumulation in the fetal liver on embryonic day 14.5 (E14.5), leading to a great reduction of hemoglobin mRNA transcription in fetal liver cells [9]. Not only QDs, other ENMs such as titanium dioxide nanoparticles and silica nanoparticles have also been demonstrated to induce adverse pregnancy complications or to cause ovarian dysfunction and led to significantly decreased fertility rate $[10,11]$. These studies highlighted that nanoparticles could adversely impact the female reproductive system. However, such studies have, to date, been confined to small animal models, and these results of nanotoxicity studies in mice would not necessarily translate to primates [12].

Cadmium selenide (CdSe) based semiconductor nanoparticles are among the most extensively studied nanomaterials, with applications ranging from energy to healthcare based on their tunable electronic and optical properties [13-15]. However, great concern has been raised regarding the potential hazards of these nanoparticles due to their cadmium content $[16,17]$. Many studies have reported toxicity of semiconductor nanoparticles in vitro and in vivo, but the results are often contradictory and inconclusive determinations of toxicity in relevance to human health has remained elusive [18, 19]. Previously, our group has reported the toxicological and pharmacological responses in male rhesus macaques after injection of $25 \mathrm{mg} \mathrm{kg}^{-1}$ of phospholipid micelle-encapsulated semiconductor nanoparticles [20]. No abnormalities were detected by measurement standard of hematological and biochemical markers based on the histological analysis of major organs. However, inductively coupled plasma mass spectroscopy (ICP-MS) analysis and fluorescence imaging showed that the nanoparticles remained in the liver, spleen, and kidneys after 90 days. Moreover, for those major organs recorded with high cadmium levels such as the spleen, liver and kidney, the presence/absence of the QD photoluminescence and the corresponding cadmium-to-selenium ratio indicated that the QDs were partially intact. This meant that the breakdown and clearance of these nanoparticles was slow. The impact of semiconductor nanoparticles on reproductive biology has been tested in rodent models, revealing that the differences in the semiconductor nanoparticle in terms of composition, surface properties, size and dosage could alter the outcome. Although semiconductor nanoparticles could cause adverse effects on the reproductive function of mice, the impact of semiconductor nanoparticles on reproductive biology of female primates was unknown [8].

In this study, nanoparticles with similar surface modification to our previous report on rhesus macaques, namely, PEGylated phospholipid micelle-encapsulated CdSe/CdS/ZnS semiconductor nanoparticles with an average hydrodynamic size of $60 \mathrm{~nm}$ were employed to probe the impacts of exposure towards semiconductor nanocrystals during pregnancy in both rodents and nonhuman primates. The biodistribution of semiconductor nanoparticles after intravenous administration in pregnant mice was extensively investigated through ICP-MS. The cadmium concentrations in the umbilical cord and the fetuses were significantly elevated after nanoparticle administration. This indicated that the semiconductor nanocrystals could traverse the placental barrier to reach the fetuses. However, we did not observe any abnormalities in the blood biomarkers of the nanocrystal treated mice, nor did we observe any irregularities in the pregnancy outcomes in the 
nanocrystal treated groups. To further assess the risk of semiconductor nanoparticles toward primate pregnancy, cynomolgus macaques were treated with the same semiconductor nanoparticle formation 14 weeks after conception. Five primates received nanoparticles at a dosage of $25 \mathrm{mg} \mathrm{kg}^{-1}$, four control monkeys were injected with buffer saline only, and two monkeys were used as absolute controls without any injections. Blood samples were collected and ultrasound scans were performed at regular time intervals before and after delivery or miscarriage. Among the experimental group, two monkeys underwent miscarriages 1 to 2 weeks following nanoparticle injection, one delivered a dead fetus 8 weeks after nanoparticle injection, and the other two delivered live offspring slightly earlier in relative to the control animals. All animals in the saline treated group and the absolute control group gave birth to live offspring.

\section{Materials and Methods}

Synthesis of CdSe quantum rods (QRs): The synthesis of CdSe quantum rods (QRs) was in accordance to a previous report [21]. Briefly, cadmium oxide (1.6 mmol), TDPA ( $3 \mathrm{mmol})$, and TOPO ( $3 \mathrm{~g})$ were loaded into a $100 \mathrm{ml}$ three-necked flask and slowly heated under an argon atmosphere of $290-300^{\circ} \mathrm{C}$. After obtaining a clear homogeneous solution, the reaction mixture was maintained at $300^{\circ} \mathrm{C}$ for another 5 minutes, then TOP-Se $(0.8 \mathrm{ml}, 1$ $\mathrm{M})$ was rapidly injected. The reaction was stopped after 2 to 3 minutes by removing the heating mantle. The QRs were separated from the surfactant solution by the addition of ethanol and centrifugation. The CdS/ZnS graded shell was formed by following the method of Manna et al [22]. Approximately $0.3 \mathrm{~g}$ of CdSe QRs was dispersed in $\sim 5 \mathrm{ml}$ toluene. Separately, $\mathrm{CdO}(2 \mathrm{mmol})$, zinc acetate $(4 \mathrm{mmol})$ and TOPO $(7 \mathrm{~g})$ were dissolved in $10 \mathrm{ml}$ oleic acid and heated to 180 ${ }^{\circ} \mathrm{C}$ for $\sim 30$ minutes under argon. The QR dispersion was injected slowly into the hot mixture, which was then held at $180{ }^{\circ} \mathrm{C}$, with a needle outlet to release evaporating toluene. The needle was removed after $\sim 15$ minutes and the temperature was raised to 210 ${ }^{\circ} \mathrm{C}$. TOP-S $(1 \mathrm{mM}, 2 \mathrm{ml})$ was then added dropwise and the mixture was held at $\sim 210{ }^{\circ} \mathrm{C}$ for 10 to15 minutes. The CdSe/CdS/ZnS QRs were then washed with ethanol and dispersed in chloroform as a stock solution. TEM images of the QRs were shown in Figure S1. It shows that the QRs were monodispersed with a rod shape and an average size of around $15 \mathrm{~nm}$ $x 5 \mathrm{~nm}$.

Phospholipid-micelle encapsulation: Chloroform dispersions of QRs $\left(\sim 4 \mathrm{mg} \mathrm{ml}^{-1}\right)$ and of DSPE-mPEG (Avanti Polar Lipids or Laysan Bio 1,2-
diacyl-sn-glycero-3-phosphoethanolamine-N-[methox $\mathrm{y}$ (polyethylene glycol)], $10 \mathrm{mg} \mathrm{ml}^{-1}$ ) were mixed in a 1:4 mass ratio and gently stirred for 5 to 10 minutes. A Labconco vacuum rotary evaporator with a water bath at room temperature was used to evaporate the chloroform. After the evaporation, the lipidic film was hydrated with 3 to $5 \mathrm{ml}$ of high-pressure liquid chromatography (HPLC) grade water and sonicated for 10 to15 minutes using a bath sonicator. The resulting dispersion was filtered through a $0.2 \mu \mathrm{m}$ syringe filter and centrifuged at 10,000 rpm to remove excess phospholipid. The precipitate was then redispersed in $2 \mathrm{ml}$ of HPLC water and kept at $4{ }^{\circ} \mathrm{C}$ for further use.

Animal studies: The study was conducted at the Laboratory Animal Center of the Academy of Military Medical Sciences, National Laboratory Primate Breeding Base, Beijing, under a protocol that was reviewed and approved by the ethics committee of Chinese PLA General Hospital (301 Hospital). BALB/c mice were obtained and handled with protocols approved by the Laboratory Animals Center of Chinese People's Liberation Army (PLA) General Hospital in Beijing. All the animal experiments and maintenance were under protocols approved by the Experimental Animal Ethics Committee of Chinese PLA General Hospital. For the studies on pregnant mice, BALB/c mice were obtained at 8 weeks of age and were housed 5 mice per cage in a 12 hour / 12 hour of light/dark cycle. Food and water were supplied ad libitum. Male and female mice were paired on a one-to-one basis and housed to mate. The show of plug was used as a marker of conception, and the day of conception was regarded as embryonic day 0 (E0). Pregnant mice were randomly grouped into two different groups as described in the text. One group of mice was divided into five subgroups (six subjects in each subgroup) and intravenously injected with semiconductor nanoparticles at a dosage of $100 \mathrm{mg} \mathrm{kg}^{-1}$ at E17. The mice were sacrificed at different time points and organ tissues were harvested for ICP-MS analysis. The other group of pregnant mice was divided into seven subgroups (six subjects in each subgroup) and was administered with buffer saline or different dosages of semiconductor nanoparticles at 25,50 and $100 \mathrm{mg}$ $\mathrm{kg}^{-1}$ at E12. Semiconductor nanoparticle treated mice were sacrificed at 2 hours or 7 days after the treatments. Tissue from the major organs and blood samples were harvested and sent for standard histological examination and ICP-MS analysis. For the studies on pregnant macaques, 25 female cynomolgus monkeys (adult) with similar menstrual cycles were selected and randomly divided into two groups. To each group, a timed mating procedure was used 
where two healthy males were placed with eight females in the breeding suite for 5 days and then replaced with another two healthy males for the next 5 days. Nine pregnant monkeys, with age ranging from 4 to 10 years and mass ranging from 3.0 to $4.2 \mathrm{~kg}$, were confirmed by ultrasonography with an Esaote Aquila Pro ultrasound scanner (Pie Medical, Maastricht, The Netherlands) after one month. The nine pregnant monkeys were randomly divided into two groups and housed in two breeding suites (five for treatment, four as control). The breeding suite contained a living room $(5.6 \mathrm{~m} \times 5.4 \mathrm{~m} \times 2.3 \mathrm{~m}, 15-23$ ${ }^{\circ} \mathrm{C}, \quad 40-80 \%$ relative humidity, $12 / 12$ hours of light/dark cycle) which was connected to a playground $(3.0 \mathrm{~m} \times 4.3 \mathrm{~m} \times 2.3 \mathrm{~m}$, with play instruments) through a door. The animals were fed with commercial monkey diets. Water was available ad libitum. Research staff inspected the monkeys three times each day. Quantum rod (10 $\left.\mathrm{mg} \mathrm{ml}^{-1}\right)$ dispersions in $0.9 \%$ sodium chloride were filter-sterilized before injection. Encapsulated quantum rods $\left(25 \mathrm{mg} \mathrm{kg}^{-1}\right)$ were intravenously administrated to five monkeys at a gestational age of 100 days. Four pregnant monkeys accepted saline injection as the control. To avoid possible impact on the pregnancy by anesthetics, the animals were held in a homemade entrapment cage where their legs could be immobilized for intravenous injection. Nanoparticle formulation or saline were injected through the vein in the left leg while the animals were not anesthetized. Before the injection, all nine monkeys were subjected to urine and blood test to provide an animal-specific baseline. Urine and fasting blood samples were collected according to the designed time points. Body mass, temperature, appearance and exploratory behavior were recorded at the same time. Also, the prenatal status (e.g., miscarriage, signs of labor, parturition) was monitored and recorded every morning. Venous blood samples $(7-8 \mathrm{ml})$ were collected in evacuated tubes containing EDTA, sodium citrate anticoagulants and non-anticoagulant agents. The blood analysis (hematology, coagulation and chemistry) was carried out on Sysmex XS-800i, Roche STA-R Evolution and Cobas 6000-C501 units, respectively. Blood samples $(0.5 \mathrm{ml})$ were taken for ICP-MS analysis using a quadrupole-based ICP-MS system (Elan DRC-II, PerkinElmer SCIEX Instruments; resolution, $\sim 0.7-0.9$ AMU). Urine (3-5 ml) was taken for urinalysis on a Roche URISYS 2400 and Sysmex UF-100i.

Histological analysis: Mice tissue from major organs were harvested and fixed with $10 \%$ buffered formalin. Monkey fetuses and placentae from miscarriage were collected while the heart, liver, spleen, lungs, kidneys, colon (section $10 \mathrm{~cm}$ in length), muscle $(5 \mathrm{~cm} \times 7 \mathrm{~cm})$, lymph nodes and brain were harvested, weighed and fixed with $10 \%$ buffered formalin. Haematoxylin and eosin-stained histological sections of the fixed organs were observed with an Olympus BX60 microscope at 40x or 20x magnification. Tissue sections were examined by two independent clinical pathologists who were not told on what kind of treatment the monkeys had undergone.

Inductively coupled plasma mass spectrometry (ICP-MS) analysis and fluorescence imaging: Tissue samples for ICP-MS analysis and fluorescence imaging were collected and kept at $-20{ }^{\circ} \mathrm{C}$. For ICP-MS, three fragments were sampled as a set from each organ of interest. Organs and sample sets were weighed before being frozen, with each set weighing $\sim 1$ g. Standard cryosectioning was performed for fluorescence imaging of the organs. Elemental concentrations were determined using a quadrupole-based ICP-MS system (Elan DRC-II, PerkinElmer SCIEX Instruments; resolution, $\sim 0.7-0.9$ AMU). Samples were digested with $\mathrm{HNO}_{3}$ and $\mathrm{HCl}$ $\left(\mathrm{HNO}_{3}: \mathrm{HCl}\right.$ ratio of $10: 1$ ) at $60^{\circ} \mathrm{C}$ for analysis.

Statistical analysis: Statistical analysis was performed using analysis of variance (ANOVA). Student's unpaired two-tailed t-test was used for any two comparisons to be obtained. A P value less than 0.01 was regarded as statistically significant. Data were presented as mean \pm SD.

\section{Results and Discussion}

The maternal and fetal circulation were separated in the placenta by the "placental barrier" which was composed of specialized blastocyst-derived trophoblast cells [23, 24]. In pregnant mice, the maturation of this barrier was found to be around the tenth day of embryonic (E10) [25]. It has been reported that fetal exposure to maternally-delivered nanoparticles was dramatically reduced after the maturation of the placental barrier [26]. In this study, pregnant BALB/C mice were intravenously treated with semiconductor nanoparticles at a dosage of $100 \mathrm{mg} \mathrm{kg}^{-1}$ before parturition on E17. Cadmium concentrations in the maternal blood, placenta, umbilical cord and fetuses at different time points of post-injection were analyzed by ICP-MS (Figure 1a). The Cd concentration in the maternal blood after an hour of semiconductor nanoparticle injection was 3,029.4 ng $\mathrm{g}^{-1}$. That concentration decreased dramatically to 647.5 ng $\mathrm{g}^{-1}$ after 24 hours. Meanwhile, the $\mathrm{Cd}$ concentration in the placenta reached $1350.1 \mathrm{ng} \mathrm{g}^{-1}$ and remained stagnant at that level. The $\mathrm{Cd}$ concentrations in the umbilical cord and fetuses in the treated group were significantly higher than that of the control. This showed that the semiconductor nanoparticles 
migrated across the mouse placental barrier and passed onto the fetuses. To test whether the placental transmigration of the injected semiconductor nanoparticles was dosage dependent, additional groups of pregnant BALB/C mice were treated with semiconductor nanoparticles on E12 at dosages of 25 $\mathrm{mg} \mathrm{kg}^{-1}, 50 \mathrm{mg} \mathrm{kg}^{-1}$ and $100 \mathrm{mg} \mathrm{kg}^{-1}$, respectively. The mice were sacrificed after 2 hours and 7 days of post-treatment while the major organs were harvested for trace elemental analysis. As shown in Figure 1b, the $\mathrm{Cd}$ concentration in the placenta increased with increasing dosage. The cadmium concentration in the placenta increased dramatically from 2 hours to 7 days of post-injection, by 365\%, 104\% and $76.9 \%$ for the dosage groups of 25,50, and $100 \mathrm{mg} \mathrm{kg}^{-1}$ groups, respectively. On the other hand, the cadmium concentration in the umbilical cord did not exhibit significant differences among the three different dosage groups after 7 days. A distinct and statistically significant difference in cadmium concentration was observed in the fetuses of the treated and control groups. The cadmium distribution in the major organs of the treated mice was shown in Figure 1c, where the liver and the spleen shared the highest cadmium concentrations. The cadmium accumulation in all of the major organs has shown similar time- and dosagedependent patterns with those in placenta. To further assess the possible toxicity of the semiconductor nanoparticle formulation, we analyzed maternal haematological and biochemical markers (Figure S2). The complete blood count (CBC) evaluations did not reveal any acute toxicity. The levels of the tested biomarkers of the semiconductor nanoparticle treated groups were within the normal range and these were comparable with those in the control group. In addition, histopathological evaluations were performed on the tissue sections of the major organs with haemotoxylin and eosin (H\&E) staining. No significant pathological changes were identified in the mice treated with semiconductor nanoparticles at three different dosages after 7 days (Figure S3).

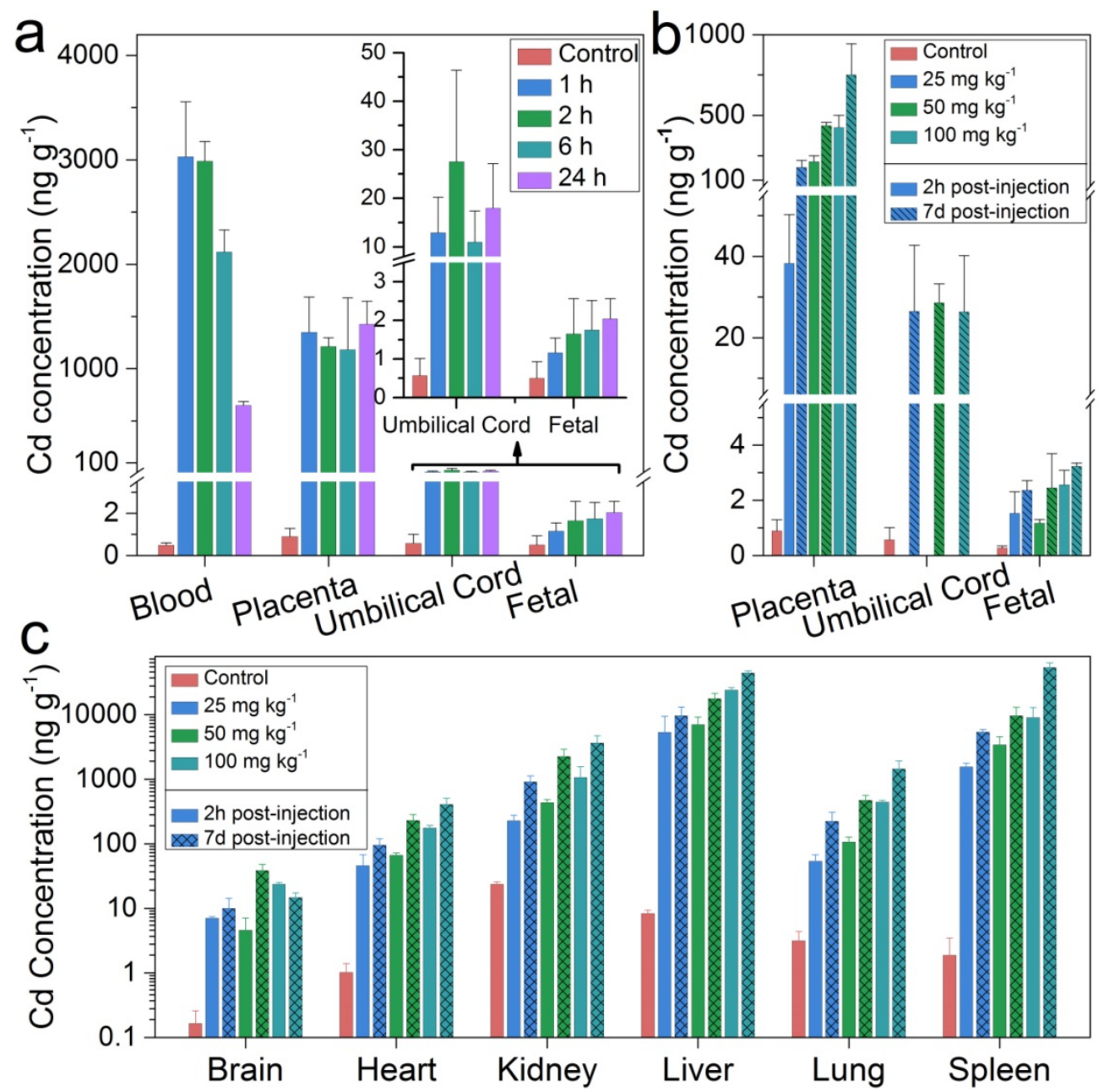

Figure 1. Cadmium (Cd) distribution in the maternal and fetal tissues of mice treated with semiconductor nanoparticles. (a) Cadmium concentrations in the maternal blood, placenta, umbilical cord and the fetuses at time points of 1, 2, 6 and $24 \mathrm{~h}$ after semiconductor nanoparticle injection at a dosage of 100 mg kg-1 on E17 $(n=6)$, in comparison with control group $(n=6)$. (b) Cd concentration in the placenta, umbilical cord and fetuses $2 \mathrm{~h}$ and 7 days after semiconductor nanoparticle injection at dosages of 25,50 and $100 \mathrm{mg} \mathrm{kg}^{-1}$ on E12 (n=6), in comparison with control group $(\mathrm{n}=6)$. (c) Cadmium distribution in the major organs of the mice in (b). 
Recently, we have demonstrated through mice that the administration of semiconductor nanocrystals two weeks before conception would not cause any pregnancy complications or significant effects of toxicity in the treated mice or their offspring [27]. That study indicated that such a buffering period after nanocrystal exposure would reduce the potential risk of the nanocrystal toward reproductive health. Here, through direct exposure during pregnancy, we have further demonstrated that the formulated semiconductor nanocrystal did not induce significant pregnancy complications in mice, even when the nanocrystals crossed the placental barriers to the pups. Moreover, in a previous study we found that intravenously administrated semiconductor nanoparticles did not cause adverse response in non-human primates (NHPs) [20]. These findings lead us to question the risks of semiconductor nanocrystal exposure during pregnancy for NHPs. Is the exposure of properly formulated semiconductor nanocrystals tolerable in NHPs alike in mice? More essentially, how should we evaluate the risks toward humans? To answer these questions, cynomolgus macaques were brought in for further evaluation. In this contribution, five cynomolgus macaques were treated with the semiconductor nanoparticle formulation at 14 weeks after conception. To minimize the potential risk to the pregnant macaques, a dosage of $25 \mathrm{mg} \mathrm{kg}^{-1}$ was used, which is at the lower end of those applied to the mice and had been proven to be relatively safe to rhesus macaques [20]. The average full gestation term of cynomolgus macaques was 167 days [28]. Thus, 14 weeks after conception corresponded to roughly 22 weeks after conception in humans [29]. At the same time, a control group of four monkeys were injected with saline buffer, while an absolute control group of two monkeys received no injections. These macaques, with body weight ranging from 3.0 to $4.8 \mathrm{~kg}$, had no exposure of nanocrystals or other exogenous agents prior to these experiments. Body masses of the pregnant monkeys were measured weekly and have shown no unusual changes after treatment. This suggested minimal systemic effects from the nanoparticle dispersion. Behavior was monitored and recorded once daily for six months. No abnormalities in eating, drinking, grooming, exploratory behavior, activity, physical features, urination or neurological status were observed. This observation was consistent with our previous finding of male rhesus macaques injected with semiconductor nanoparticles.

Ultrasound scans were used to monitor the uterus and fetal development in the gestational sac (Figure S4, a) at 4, 8 and 14 weeks after conception. At these time points, prior to nanoparticle treatment, fetal development was normal in all cases. In a typical scan, the fetus was observed to grow normally with a crown-rump length of 7.32 and $10.7 \mathrm{~cm}$ at week 8 and 14 (Figure S4, b, c), respectively. These values were in good agreement with the reported normal range of the crown-rump length of a healthy developing fetus [30]. However, ultrasound analysis after injection of semiconductor nanocrystals revealed slight abnormalities in the development of fetuses in terms of heart rate, reduced movements and slower development, as confirmed by two radiologists. In one case, we did not detect any heart rate from the fetus (Figure S4, d). Eventually, for that case, a miscarried fetus was delivered 8 weeks after the nanoparticle treatment. In two other cases, miscarriage happened on the first and third day after the nanoparticle treatment. The uteruses of the monkeys that miscarried were also monitored and comparable lateral lengths of uterus were determined for both experimental and control subjects (Figure S4, $e, f)$.

Due to the minimal cadmium exposure toward macaques under normal conditions, the background levels of cadmium in the tissues were extremely low. The biodistribution of semiconductor nanoparticles in the placenta and miscarried fetuses were examined by inductively coupled plasma mass spectroscopy (ICP-MS) analysis. Certain amounts of cadmium traces were found in the placenta, umbilical cord, and fetal liver, spleen, and kidney, where the average concentrations were 486, 24.5, 14.8, 7.7, and $13.2 \mathrm{ng} \mathrm{g}^{-1}$, respectively (Figure 2a). Low levels (below $4 \mathrm{ng} \mathrm{g}^{-1}$ ) were observed in the heart, lung and spleen of the miscarried fetus. Nonetheless, the detected cadmium levels were still significantly higher than those observed in the tissues collected from miscarried fetuses from the general population of monkeys in the animal research center which served as the control for this measurement. In addition to analyzing the major organs, the overall cadmium level in the miscarried fetuses was evaluated. In our study, $25 \mathrm{mg} \mathrm{kg}^{-1}$ of the semiconductor nanoparticle formulation corresponded to $0.815 \mathrm{mg} \mathrm{kg}^{-1}$ of cadmium. A careful evaluation found that approximately $0.16 \%$ of the initial dose of cadmium was transferred to the fetus. Although the semiconductor nanoparticle formulation contained other elements including selenium, sulfur and zinc, the background levels of these elements in the tissues were higher than the contribution of the semiconductor nanoparticles. As a result, no significant difference was observed in the levels of these elements between the semiconductor nanoparticle treated animals and the control. The blood clearance profile in the mothers was also determined (Figure $2 b$ ). Twenty-four hours after the injection, the concentrations of cadmium in the blood 

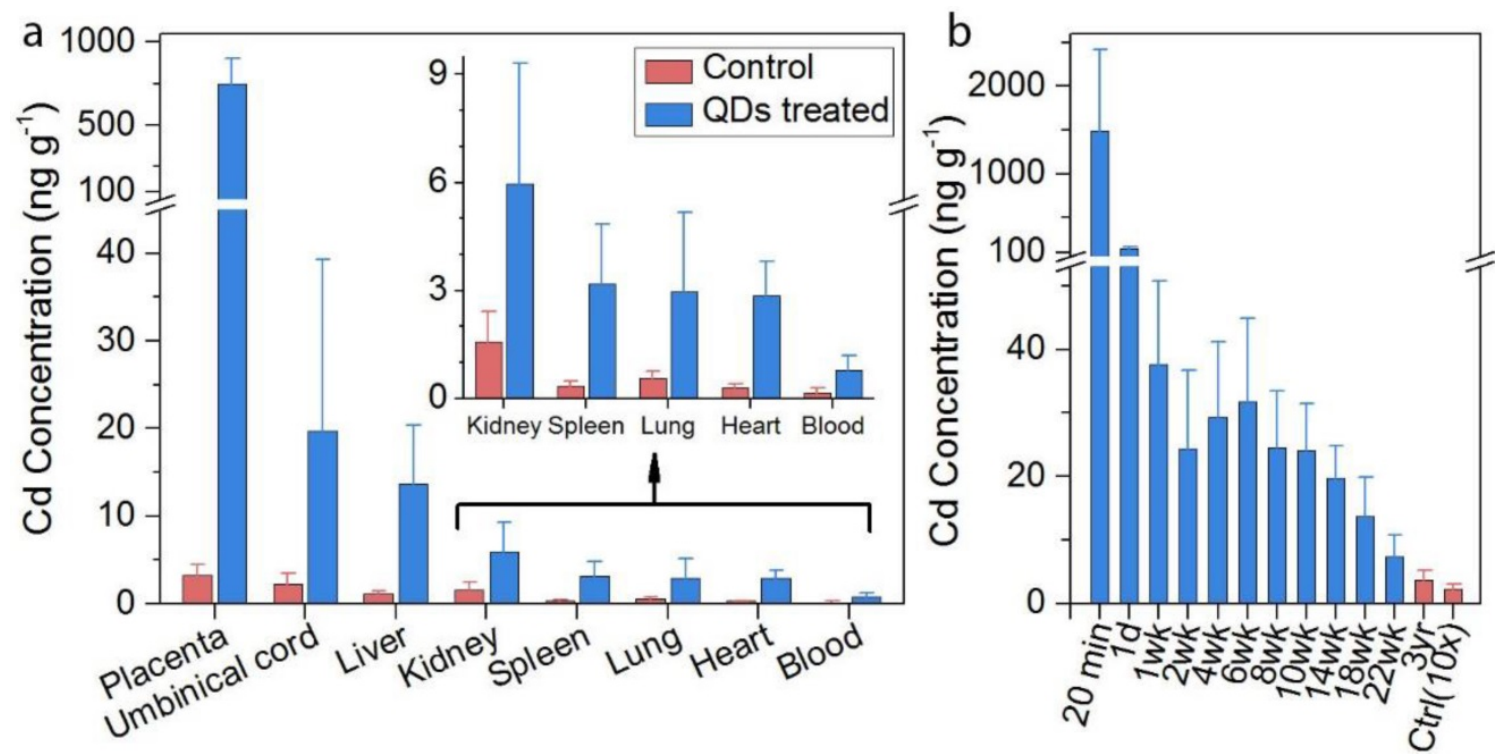

Figure 2. (a) In vivo biodistribution of cadmium in the fetal major organs of treated animals after miscarriage ( $\mathrm{n}=3$, blue bars) as compared with background control $(n=5$, red bars). The control miscarried fetuses were obtained from natural miscarriages in the animal center. (b) Blood cadmium concentration profiles after injection of treated animals ( $n=5$, blue bars) in comparison to the background control ( $n=9$, red bar, multiplied by 10).

decreased to $148.3 \mathrm{ng} \mathrm{ml}{ }^{-1}$. The concentration of cadmium in the blood fell below $40 \mathrm{ng} \mathrm{ml}^{-1}$ after 1 week and continued to drop gradually afterwards. It should be noted that, after 3-years of monitoring, the blood cadmium concentration remained at $3.74 \mathrm{ng}$ $\mathrm{ml}^{-1}$ in the semiconductor nanoparticle treated group, which was significantly higher than the value of 0.23 $\mathrm{ng} \mathrm{ml}{ }^{-1}$ in the control group. In addition to ICP-MS analysis, we have used fluorescence imaging to identify intact semiconductor nanoparticles in tissues. However, the semiconductor nanoparticle concentration was too low to enable direct imaging approach for semiconductor nanoparticle quantification in the fetal organs. Thus, ICP-MS analysis is a more reliable and practical approach to quantify the biodistribution of nanoparticles in the miscarried fetuses.

Histological analysis was performed on tissues from the placenta, umbilical cord and miscarried fetuses (Figure 3). Control fetuses obtained from miscarriages occurring in the general population of the primate center were also subjected to histopathological examinations for comparison (Supplementary Figs. S5, S6 and S7). No pathological changes in the placenta and umbilical cord of the miscarried fetuses were observed. The miscarried fetal heart, lung, kidney, liver, and spleen also exhibited no abnormalities. No sign of inflammatory response or injury was observed in the liver samples. The lung samples did not reveal any sign of pulmonary fibrosis. The glomerulus structure in the kidney section could be observed without any difficulty. As confirmed by two experienced pathologists, no signs of necrosis were detected in any of the examined histological samples.

The overall miscarriage rate of $\sim 800$ female cynomolgus monkeys in the National Laboratory Primate Breeding Base was estimated to be $15 \%$, including miscarriages occurring before week 14 . However, the gestational age of miscarriage was not known for all of the samples, and we therefore took $15 \%$ as an upper limit for the spontaneous miscarriage rate. The $60 \%$ miscarriage rate among our treated animals was a four-fold increase over this spontaneous miscarriage rate, and much more than a four-fold increase over the post-14 week spontaneous miscarriage rate. Assuming a spontaneous miscarriage rate of $15 \%$, the probability of 3 or more out of 5 experimental animals underwent spontaneous miscarriage was $2.7 \%$. Thus, even with this modest number of animals $(n=5$ in the treated group), the probability that the effect was real still would be more than $97 \%$. Many nonchromosomal factors were associated with miscarriage, including endocrine abnormalities, anatomic abnormalities, inherited, acquired thrombophilia, environmental exposures and immunologic factors. Despite this long list of associated factors, detailed understanding of miscarriage remained limited [31, 32]. Histopathology is regarded as the gold standard for diagnosis. However, our histological analysis did not reveal any cellular-level damage to the placenta or abnormal development of the miscarried fetuses. To identify the cause of the abnormal outcome in this study, the maternal haematological and biochemical markers were monitored during the whole experimental 


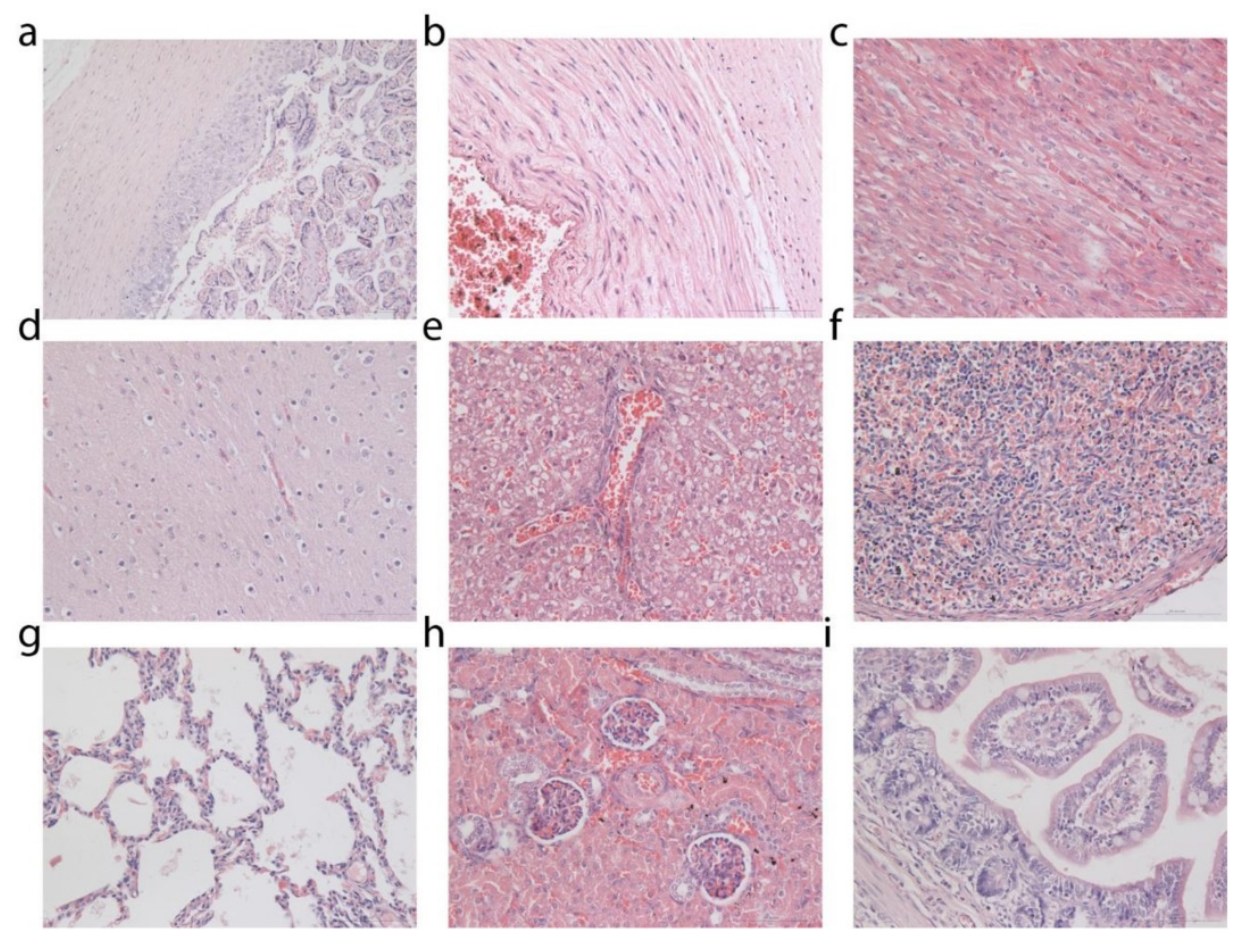

Figure 3. Histological analysis of placenta and miscarried fetuses. Tissues were collected from placenta (a), umbilical cord (b), heart (c), brain (d), liver (e), spleen (f), lung (g), kidney (h) and intestine (i). Two clinical pathologists, both unaware of whether the tissues came from the treated or control fetuses, analyzed tissue sections and found no abnormalities. Histological images of fetal tissue sections from two additional treated monkeys and one miscarriage from an untreated animal of the general primate center population are provided in Supplementary Figures.

been caused by the associated inflammation. However, these biomarkers reverted to their normal range within a week, indicating recovery from the hepatocellular injury. It should be noted that the two cases of miscarriage occurred within three days after the administration of the semiconductor nanoparticle formulation. This suggested that the injection of semiconductor nanoparticle formulation had induced the acute hepatocellular injury and when combined with possible stress caused by the injection, these joint effects had eventually led to a high miscarriage rate.

To further probe whether the impact of the semiconductor nanoparticles on the primate reproductive system was permanent, the five treated monkeys

course (Figure 4). The complete blood count (CBC) evaluations did not reveal any acute toxicity (Figure $4 \mathrm{~b}-\mathrm{i})$. The injected nanocrystal formulation did not induce any significant changes in the levels of haemoglobin $(\mathrm{Hb})$, hematocrit (Hct), alkaline phosphatase (ALP), total protein (TPROT), albumin (ALB), total bilirubin (TBILI), direct bilirubin (DBIL), gamma glutamyl transaminase $(\gamma-G T)$, blood urea nitrogen (BUN) or creatinine (CRE) (Figure 4a, j-q, r and $\mathrm{t}-\mathrm{u}$ ). This indicated that the nanocrystals did not cause maternal kidney damage at the administered dose of $25 \mathrm{mg} \mathrm{kg}^{-1}$. Also, triglycerides (TG), blood glucose (GLU) and cholesterol ( $\mathrm{CHO}$ ) were within the physiological range (Figure $4 \mathrm{v}-\mathrm{x}$ ). For all the examined biomarkers, the only variation observed was a raise in the alanine transaminase (ALT), aspartate transaminase (AST) and C-reactive protein (CRP) levels one week after the injection (Figure $4 \mathrm{p}, \mathrm{q}$ and s). Serum ALT is the clinical chemistry gold standard of hepatotoxicity and AST is also associated with liver toxicity [33]. C-reactive protein (CRP) is a phylogenetically highly conserved plasma protein that participates in the systemic response to inflammation. Its plasma concentration increases during inflammatory states, which has long been employed for clinical purposes [34]. The increase in ALT and AST values is an indication of acute hepatocellular injury. The elevation in CRP may have including the three that experienced miscarriages were allowed to mate again after they had fully recovered. Three of these five monkeys, including two who have experienced miscarriage after semiconductor nanocrystal injection, were successfully impregnated and delivered healthy offspring. No miscarriage was observed for this set of experiments. No abnormality in eating, exploratory behavior, activity, or neurological status was observed for the newborn monkeys. The mothers and newborns underwent continued evaluation and have shown no ill effects for over 2 years to date. ICP-MS analysis was performed on the placental and umbilical cord tissues to determine the cadmium levels. The average cadmium concentrations in the placenta and umbilical cord were found to be 39.06 and $10.60 \mathrm{ng} \mathrm{g}^{-1}$, respectively. These were considerably lower than those in the first pregnancy of the semiconductor nanoparticle treated group, yet still higher than those in the control group. This was not surprising, knowing that the blood cadmium concentration remained well above the normal range in the semiconductor nanoparticle treated monkeys. Nonetheless, these results suggested that the impact of the semiconductor nanoparticles in increasing the miscarriage risk in primate pregnancy was not a long-lasting or permanent effect. 

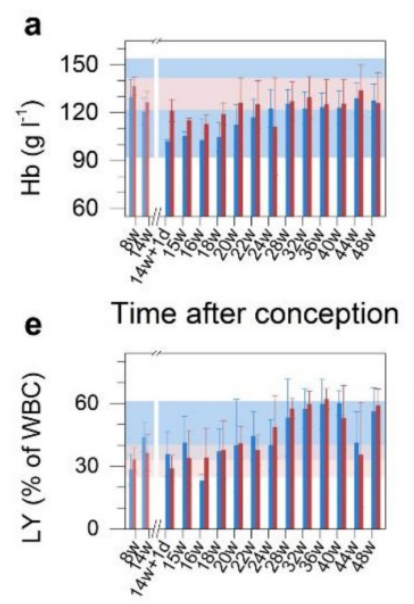

i Time after conception

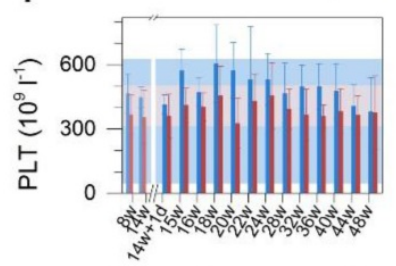

m Time after conception
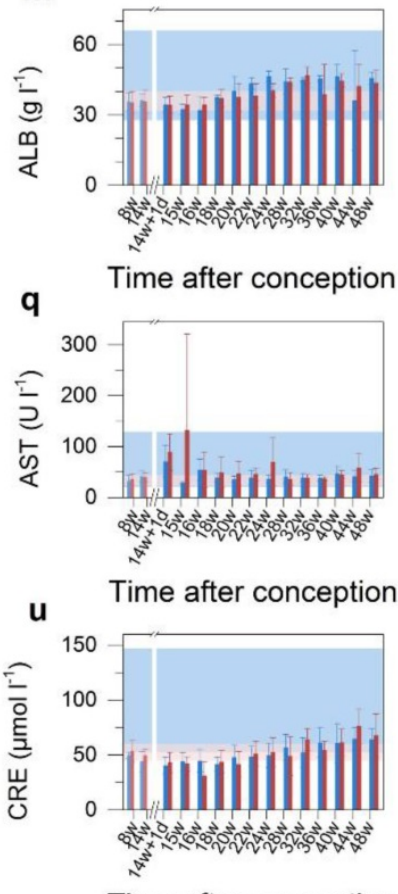

Time after conception

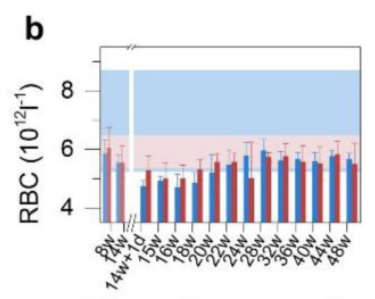

Time after conception

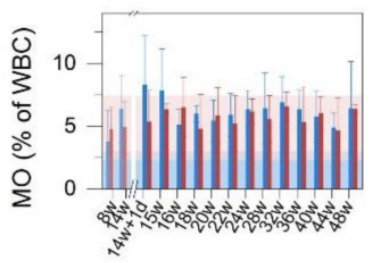

Time after conception

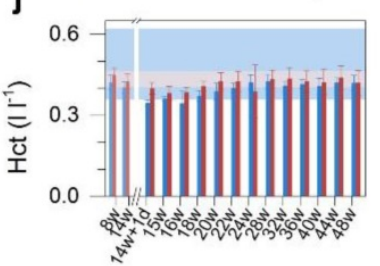

Time after conception

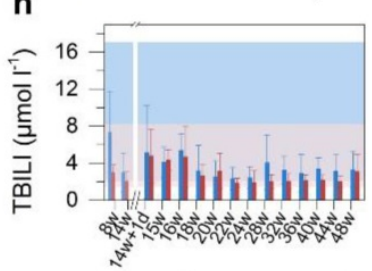

Time after conception

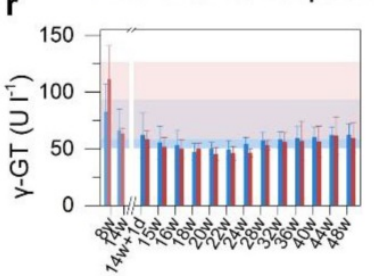

Time after conception

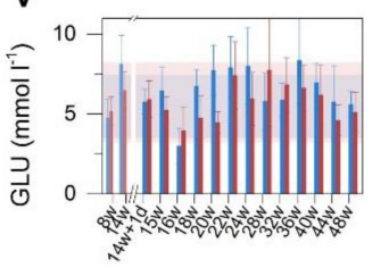

Time after conception
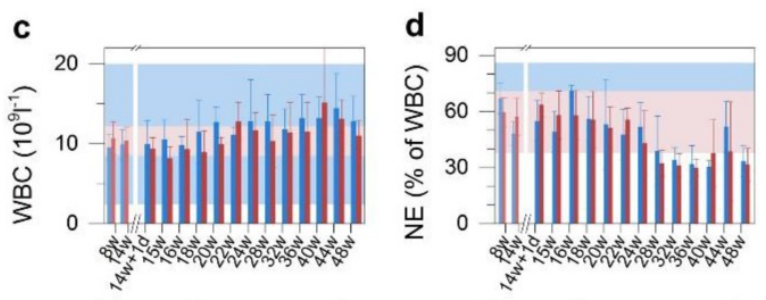

g Time after conception
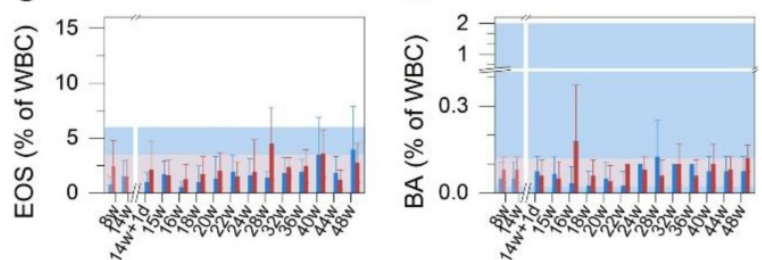

k Time after conception

Time after conception
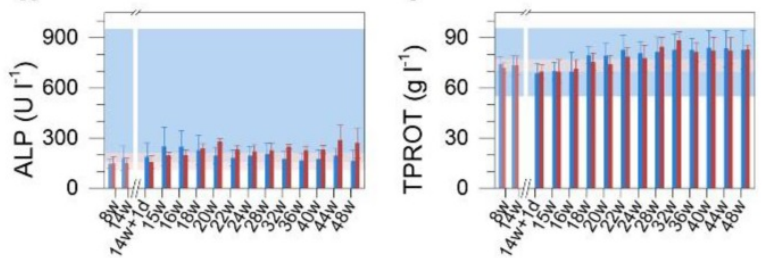

Time after conception

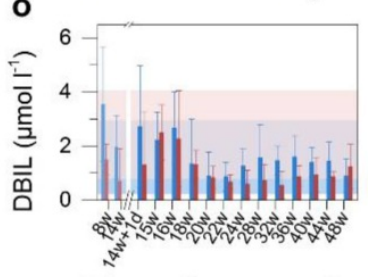

Time after conception
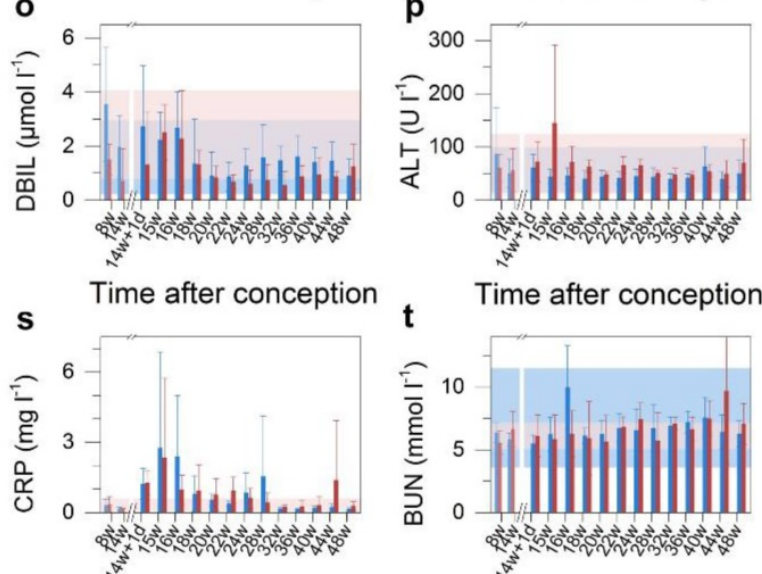

t Time after conception

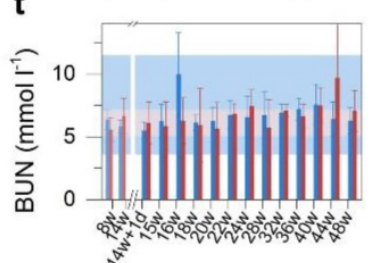

w Time after conception
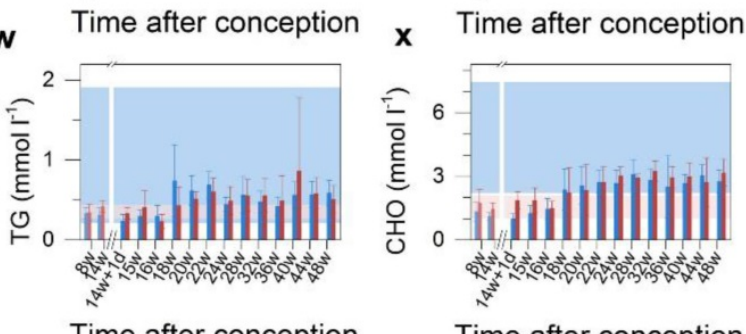

Time after conception

Figure 4. Blood test results for treated Macaca fascicularis after conception. $a-x$, The results of treated animals ( $n=5$, red) show no significant abnormalities in immune response, kidney or liver function, blood clotting or blood chemistry when compared to the control group receiving saline buffer ( $\mathrm{n}=4$, blue) over the 280 day period. Time of conception is used as the reference. Error bars represent one standard deviation. The region rendered in blue represents the normal range from the literature, and the pink region represents the range observed in the test subjects before treatment $(n=9)$. A break after 14 weeks indicates the time of treatment. Abbreviations: haemoglobin, Hb; red blood cell count, RBC; white blood cell count, WBC; neutrophil granulocyte, NE; lymphocyte, LY; monocyte, MO; eosinophil granulocyte, EOS; basophil granulocyte, BA; platelet count, PLT; haematocrit, Hct; alkaline phosphatase, ALP; total protein, TPROT; albumin, ALB; total bilirubin, TBILI; direct bilirubin, DBIL; alanine transaminase, ALT; aspartate transaminase, AST; gamma glutamyl transferase, $\mathrm{Y}$-GT; C-reactive protein, CRT; blood urea nitrogen, BUN; creatinine, CRE; blood glucose, GLU; triglyceride, TG; total cholesterol, CHO.

The placenta is an organ that is required for maternal-fetal exchange and mandatory for preservation of gestation as well as fetus growth.
Recent small animal studies suggested that the placenta was not an effective barrier for preventing transfer of nanoscale agents. Nanoparticles such as 
gold nanoparticles [35], titanium dioxide nanoparticles [36], silica nanoparticles [11], semiconductor nanoparticles (e.g. quantum dots) [8], and carbon nanoparticles [37], were able to migrate across the placental barrier of small animals. Nanoparticles of smaller sizes were more likely to migrate across the placenta. For instance, when $1.4 \mathrm{~nm}$ and $18 \mathrm{~nm}$ diameter gold nanoparticles were injected into pregnant rats, a higher concentration of $1.4 \mathrm{~nm}$ nanoparticles was found in the placenta compared to the $18 \mathrm{~nm}$ particles [38]. Also, when 70, 300 and 3000 $\mathrm{nm}$ silica particles were injected into pregnant BALB/c mice, only the $70 \mathrm{~nm}$ nanoparticles were observed in the placenta and the fetal major organs [11]. In addition to nanoparticle size, surface properties of the nanoparticles appeared to play a vital role in determining the biodistribution in vivo [39]. The ability of semiconductor nanoparticles to cross over the placenta in mice has been shown to reduce after modifying their surface with polyethylene glycol (PEG) or with a silica shell. Here, we somewhat surprisingly found that even PEGylated micelle-encapsulated semiconductor nanoparticles crossed the placenta of treated mice, in the absence of any functional groups. Nanoparticles that crossed the placental barrier have been shown to induce both structural and functional abnormalities in the mouse placenta and thus caused pregnancy complications. Multiple factors, such as placental cellular damage, coagulation, complement activation and oxidative stress induced by nanoparticles in the placenta were suggested as possible causes of these complications [11]. Nanoparticle-generated reactive oxygen species (ROS) have also been identified as important contributors to female reproductive and developmental toxicity in mice [40]. However, in this contribution, although the semiconductor nanocrystals crossed the placenta, no significant pregnancy complication was observed in the treated groups of mice. We believed the PEGylation in the nanoparticle formulation may have greatly reduced the binding affinities of proteins toward the nanomaterials and thus reduced their fetal toxicity.

Although mouse models were often adopted for toxicology studies and the results were used as references for clinical trials, systematic studies have shown that responses in mouse models of diseases could correlate poorly with responses in humans [41, 42]. In contrast, the cynomolgus macaques were much more closely related to humans than rodents and have been widely recognized as an important nonhuman primate model in drug development, drug testing, and toxicology [43-45]. They were highly responsive to external stress, and thus have been employed to study the effects of stress on reproductive function as well as interactions of estrogens with dietary and external risk factors for diseases [46-48]. Particularly in the study of placentation, macaques shared close similarities with humans in terms of the villous structure, nature of the interhaemal barrier, and pattern of circulation in the intervillous space [49]. In this contribution, while different outcomes have been observed for mice and macaques when they were exposed to semiconductor nanocrystal during pregnancy, we believed that the observation of increased miscarriage risk in macaques was more predictive of possible responses in humans.

\section{Conclusion}

In summary, semiconductor nanocrystals encapsulated within PEGylated phospholipid micelles were observed to cross the placenta during pregnancy in both mice and macaques. However, the responses of the mice and macaques to the semiconductor nanocrystals were quite different. For mice treated with the CdSe/CdS/ZnS semiconductor nanocrystals during pregnancy at dosages of 25,50 or $100 \mathrm{mg} \mathrm{kg}^{-1}$, no significant abnormality was observed in blood biomarkers, histopathology or behavior. On the other hand, the macaque group showed an increased rate of miscarriage when intravenously injected with $25 \mathrm{mg} \mathrm{kg}^{-1}$ of the semiconductor nanocrystal formulation 14 weeks after conception. The nanoparticles were found in the placenta and major organs of the miscarried fetuses. Despite the fact that no pathological change was identified in the placenta or major organs of the miscarried fetuses, our data suggested that the PEGylated micelleencapsulated nanoparticles have high potential for inducing miscarriage in primates, with at least a four-fold increase in miscarriage rate among treated animals. This was relative to the spontaneous miscarriage rate among the general population in the same animal facility. An acute hepatocellular injury induced by the administrated semiconductor nanoparticle formulation has been suggested as a major factor in the elevated miscarriage rate. Based on this first study of comparing semiconductor nanocrystal toxicity between pregnant mice and nonhuman primates, we suggested that precautions must be required to minimize the impact on reproductive function of those who may be exposed to engineered nanoparticles during pregnancy. However, it should also be noted that the impact of nanoparticles on the pregnant monkeys was not permanent, as the monkeys experiencing nanoparticle-induced miscarriage were subsequently able to deliver healthy offspring. 


\section{Supplementary Material}

Supplementary figures and tables. http://www.ntno.org/v03p0054s1.pdf

\section{Acknowledgements}

This work was supported by the National Natural Science Foundation of China (No. 51371197, 31728008), Singapore Ministry of Education (Grants Tier 2 MOE2010-T2-2-010 (M4020020.040 ARC2/11) and Tier 1 M4010360.040 RG29/10), NTU-NHG Innovation Collaboration Grant (No. M4061202.040), and $\mathrm{A}$ *STAR Science and Engineering Research Council (No. M4070176.040).

\section{Competing Interests}

The authors have declared that no competing interest exists.

\section{References}

1. Oliver J. Quantum dots: global market growth and future commercial prospects: BCC Research; 2016.

2. Kim JY, Voznyy O, Zhitomirsky D, Sargent EH. 25th Anniversary Article: Colloidal Quantum Dot Materials and Devices: A Quarter-Century of Advances. Advanced Materials. 2013; 25: 4986-5010.

3. Shirasaki Y, Supran GJ, Bawendi MG, Bulovic V. Emergence of colloidal quantum-dot light-emitting technologies. Nature Photonics. 2013; 7: 13-23.

4. De Matteis V, Rinaldi R. Toxicity Assessment in the Nanoparticle Era. In: Saquib Q, Faisal M, Al-Khedhairy AA, Alatar AA, editors. Cellular and Molecular Toxicology of Nanoparticles. Cham: Springer International Publishing; 2018. p. 1-19.

5. Grandjean P, Landrigan PJ. Developmental neurotoxicity of industrial chemicals. The Lancet. 2006; 368: 2167-78

6. Brohi RD, Wang L, Talpur HS, Wu D, Khan FA, Bhattarai D, et al. Toxicity of Nanoparticles on the Reproductive System in Animal Models: A Review. Frontiers in Pharmacology. 2017; 8.

7. Ema M, Kobayashi N, Naya M, Hanai S, Nakanishi J. Reproductive and developmental toxicity studies of manufactured nanomaterials. Reproductive Toxicology. 2010; 30: 343-52.

8. Chu M, Wu Q, Yang H, Yuan R, Hou S, Yang Y, et al. Transfer of Quantum Dots from Pregnant Mice to Pups Across the Placental Barrier. Small. 2010; 6: 670-8.

9. Wang Z, Liu W, Zhang S, Zhang J, Liu S. CdSe Quantum Dots Incurred Hemoglobin RNA Transcription Inhibition in Embryonic Erythroid Precursors and Compromised Embryonic Development in Mice under Low-Dose Exposure. ACS Sustainable Chemistry \& Engineering. 2018; 6: 4164-73.

10. Gao G, Ze Y, Li B, Zhao X, Zhang T, Sheng L, et al. Ovarian dysfunction and gene-expressed characteristics of female mice caused by long-term exposure to titanium dioxide nanoparticles. Journal of Hazardous Materials. 2012; 243: 19-27.

11. Yamashita K, Yoshioka Y, Higashisaka K, Mimura K, Morishita Y, Nozaki $M$, et al. Silica and titanium dioxide nanoparticles cause pregnancy complications in mice. Nat Nano. 2011; 6: 321-8.

12. Liu J, Erogbogbo F, Yong K-T, Ye L, Liu J, Hu R, et al. Assessing Clinical Prospects of Silicon Quantum Dots: Studies in Mice and Monkeys. ACS Nano. 2013; 7: 7303-10.

13. Bawendi MG, Steigerwald ML, Brus LE. The Quantum Mechanics of Larger Semiconductor Clusters ("Quantum Dots"). Annual Review of Physical Chemistry. 1990; 41: 477-96.

14. Kamat PV. Quantum Dot Solar Cells. Semiconductor Nanocrystals as Light Harvesterst. The Journal of Physical Chemistry C. 2008; 112: 18737-53.

15. Medintz IL, Uyeda HT, Goldman ER, Mattoussi H. Quantum dot bioconjugates for imaging, labelling and sensing. Nat Mater. 2005; 4: $435-46$.

16. Derfus AM, Chan WCW, Bhatia SN. Probing the Cytotoxicity of Semiconductor Quantum Dots. Nano Letters. 2003; 4: 11-8.
17. Fitzpatrick JAJ, Andreko SK, Ernst LA, Waggoner AS, Ballou B, Bruchez MP. Long-term Persistence and Spectral Blue Shifting of Quantum Dots in Vivo. Nano Letters. 2009; 9: 2736-41.

18. Yong K-T, Law W-C, Hu R, Ye L, Liu L, Swihart MT, et al. Nanotoxicity assessment of quantum dots: from cellular to primate studies. Chemical Society Reviews. 2013; 42: 1236-50.

19. Wang $Y$, Tang M. Review of in vitro toxicological research of quantum dot and potentially involved mechanisms. Science of The Total Environment. 2018; 625: 940-62.

20. Ye L, Yong K-T, Liu L, Roy I, Hu R, Zhu J, et al. A pilot study in non-human primates shows no adverse response to intravenous injection of quantum dots. Nat Nano. 2012; 7: 453-8

21. Yong K-T, Qian J, Roy I, Lee HH, Bergey EJ, Tramposch KM, et al. Quantum Rod Bioconjugates as Targeted Probes for Confocal and Two-Photon Fluorescence Imaging of Cancer Cells. Nano Letters. 2007; 7: 761-5.

22. Manna L, Scher EC, Li L-S, Alivisatos AP. Epitaxial growth and photochemical annealing of graded CdS/ZnS shells on colloidal CdSe nanorods. Journal of the American Chemical Society. 2002; 124: 7136-45.

23. Jansson T, Powell Theresa L. Role of the placenta in fetal programming: underlying mechanisms and potential interventional approaches. Clinical Science. 2007; 113: 1.

24. Leiser R, Kaufmann P. Placental structure: in a comparative aspect. Exp Clin Endocrinol Diabetes. 1994; 102: 122-34.

25. Watson ED, Cross JC. Development of Structures and Transport Functions in the Mouse Placenta. Physiology. 2005; 20: 180.

26. Yang H, Sun C, Fan Z, Tian X, Yan L, Du L, et al. Effects of gestational age and surface modification on materno-fetal transfer of nanoparticles in murine pregnancy. Scientific Reports. 2012; 2: 847.

27. Liu J, Yang C, Liu J, Hu R, Hu Y, Chen H, et al. Effects of Cd-based Quantum Dot Exposure on the Reproduction and Offspring of Kunming Mice over Multiple Generations. Nanotheranostics. 2017; 1: 23-37.

28. Wolfensohn S. Old World Monkeys. The UFAW Handbook on the Care and Management of Laboratory and Other Research Animals: Wiley-Blackwell; 2010. p. 592-617

29. Patel RR, Steer P, Doyle P, Little MP, Elliott P. Does gestation vary by ethnic group? A London-based study of over 122000 pregnancies with spontaneous onset of labour. International Journal of Epidemiology. 2004; 33: 107-13.

30. Chellman GJ, Bussiere JL, Makori N, Martin PL, Ooshima Y, Weinbauer GF. Developmental and reproductive toxicology studies in nonhuman primates. Birth Defects Research Part B: Developmental and Reproductive Toxicology. 2009; 86: 446-62.

31. Brown S. Miscarriage and Its Associations. Semin Reprod Med. 2008; 26: 391-400.

32. García-Enguidanos A, Calle ME, Valero J, Luna S, Domínguez-Rojas V. Risk factors in miscarriage: a review. European Journal of Obstetrics \& Gynecology and Reproductive Biology. 2002; 102: 111-9.

33. Ozer J, Ratner M, Shaw M, Bailey W, Schomaker S. The current state of serum biomarkers of hepatotoxicity. Toxicology. 2008; 245: 194-205.

34. Black S, Kushner I, Samols D. C-reactive Protein. Journal of Biological Chemistry. 2004; 279: 48487-90.

35. Takahashi S, Matsuoka O. Cross placental transfer of 198Au-colloid in near term rats. Journal of radiation research. 1981; 22: 242-9.

36. Shi H, Magaye R, Castranova V, Zhao J. Titanium dioxide nanoparticles: a review of current toxicological data. Part Fibre Toxicol. 2013; 10: 15.

37. Campagnolo L, Massimiani M, Palmieri G, Bernardini R, Sacchetti C, Bergamaschi A, et al. Biodistribution and toxicity of pegylated single wall carbon nanotubes in pregnant mice. Particle and fibre toxicology. 2013; 10: 21.

38. Semmler-Behnke M, Fertsch S, Schmid G, Wenk A, Kreyling WG. Uptake of $1.4 \mathrm{~nm}$ versus $18 \mathrm{~nm}$ gold nanoparticles in secondary target organs is size dependent in control and pregnant rats after intratracheal or intravenous application. EuroNanoForum 2007. 2007: 102.

39. Albanese A, Tang PS, Chan WCW. The Effect of Nanoparticle Size, Shape, and Surface Chemistry on Biological Systems. Annual Review of Biomedical Engineering. 2012; 14: 1-16.

40. Sun J, Zhang Q, Wang Z, Yan B. Effects of nanotoxicity on female reproductivity and fetal development in animal models. International journal of molecular sciences. 2013; 14: 9319-37.

41. Breschi A, Gingeras TR, Guigo R. Comparative transcriptomics in human and mouse. Nat Rev Genet. 2017; 18: 425-40.

42. Rice J. Animal models: Not close enough. Nature. 2012; 484: S9-S.

43. Fouchier RAM, Kuiken T, Schutten M, van Amerongen G, van Doornum GJJ, van den Hoogen BG, et al. Aetiology: Koch's postulates fulfilled for SARS virus. Nature. 2003; 423: 240-.

44. Leonard JP, Sherman ML, Fisher GL, Buchanan LJ, Larsen G, Atkins MB, et al. Effects of single-dose interleukin-12 exposure on 
interleukin-12-associated toxicity and interferon- $\gamma$ production. Blood. 1997; 90: 2541-8.

45. Sullivan NJ, Sanchez A, Rollin PE, Yang Z-y, Nabel GJ. Development of a preventive vaccine for Ebola virus infection in primates. Nature. 2000; 408: 605-9.

46. Bethea CL, Centeno ML, Cameron JL. Neurobiology of stress-induced reproductive dysfunction in female macaques. Molecular neurobiology. 2008; 38: 199-230

47. Manuck SB, Kaplan JR, Adams MR, Clarkson TB. Effects of stress and the sympathetic nervous system on coronary artery atherosclerosis in the cynomolgus macaque. American Heart Journal. 1988; 116: 328-33.

48. O'Brien T, Wagner J, Litwak K, Carlson C, Cefalu W, Jordan K, et al. Islet amyloid and islet amyloid polypeptide in cynomolgus macaques (Macaca fascicularis): an animal model of human non-insulin-dependent diabetes mellitus. Veterinary Pathology Online. 1996; 33: 479-85.

49. Carter AM. Animal Models of Human Placentation - A Review. Placenta. 2007; 28: S41-S7. 